\title{
Uma análise sobre a abordagem institucional de Hayek e alguns de seus conceitos
}

\author{
Eduardo Angeli \\ Professor - Universidade Federal do Paraná (UFPR) \\ Endereço: Av. Prefeito Lothário Meissner, 632 - Jardim Botânico - Curitiba - Paraná \\ CEP: 80210-170 - E-mail: eduardoangeli@hotmail.com
}

Recebido: 10/02/2016. Aceite: 20/04/2017.

\section{Resumo}

O objetivo do artigo é contribuir com o estudo da abordagem institucional de Hayek ao expor uma classificação de alguns dos conceitos institucionais hayekianos. Para se alcançar tal intento, é argumentado que o estudo de Hayek sobre temas institucionais pode ser visto como uma consequência da preocupação do austríaco em explorar o problema do conhecimento e de coordenação existente na sociedade, bem como um elemento a enriquecer sua crítica ao racionalismo construtivista - com isso, é feito com que o institucionalismo hayekiano se encaixe na trajetória de pesquisa do autor. A seguir, passa-se a uma análise de conceitos institucionais hayekianos, explicando de modo sumário o que o austríaco entendia por ordem, lei, legislação e regras, e propondo uma divisão deste último conceito em regras de conduta, regras de conhecimento tácito, e regras mentais.

\section{Palavras-Chave}

F. A. Hayek. Instituições. Regras.

\begin{abstract}
The paper aims to contribute to the study of Hayek's institutional approach. For that, it is proposed a classification of some of the Hayekian institutional concepts. Additionally, it is argued that Hayek's focus on institutional issues can be understood as a consequence of his intention to investigate the problem of knowledge and coordination in society, as well as an element to enrich his critique of constructivist rationalism. Then it is made an analysis of some Hayekian institutional concepts, briefly explaining what was his understanding about order, law, legislation and rules, and proposing a classification of the latter concept in rules of conduct, tacit rules, and mental rules.
\end{abstract}

\section{Keywords}

F. A. Hayek. Institutions. Rules.

\section{JEL Classification}

B25. B31. B53.

- Agradeço, pelas valiosas críticas e sugestões, a David Dequech, Fabio Barbieri, Mauricio Coutinho, Ricardo Feijó e Rogerio Pereira de Andrade, além de dois pareceristas anônimos da Estudos Econômicos. Agradeço também à Capes e ao CNPq pelo apoio financeiro. Os erros e omissões são de minha inteira responsabilidade. 


\section{Introdução}

O presente artigo pretende contribuir com o estudo da abordagem institucional de F. A. Hayek ao propor uma classificação de alguns dos conceitos institucionais hayekianos: regras (divididas em três tipos), ordem, lei e legislação. Para alcançar tal intento, é apresentado o papel que as instituições possuem no programa de pesquisa de Hayek.

Hayek, ao longo de seis décadas de intensa atividade intelectual, publicou dezenas de livros e centenas de artigos (Boettke 2000). A vastidão de sua obra, além da grande gama de temas por ele abordados, têm suscitado o trabalho, por parte de diversos historiadores do pensamento econômico, de sistematização e reinterpretação de seu pensamento e de suas contribuições. O’Driscoll (1977), Gray (1984), Fleetwood (1995) e Caldwell (2004) são exemplos de importantes livros que bem representam tal tipo de exercício. Boettke (2000), Wood e Woods (1991), Frowen (1997), Feser (2006) e Garrison e Barry (2014) representam trabalhos de fôlego de organização de artigos acadêmicos de reflexão a respeito da obra hayekiana. Diante disso, o presente artigo pretende contribuir com tal esforço para o aprofundamento da compreensão acerca da contribuição hayekiana. Em particular, o texto explica o papel que o estudo das instituições desempenha na obra de Hayek, apresenta a concepção de Hayek para alguns termos pertinentes à análise institucional, e propõe uma classificação para variantes de um destes conceitos, qual seja, o de regras.

Após esta introdução, o artigo está dividido em duas seções. Na seção 2, argumenta-se que, tendo por pano de fundo o debate do cálculo econômico socialista, o estudo de Hayek sobre temas institucionais pode ser visto como uma consequência da preocupação do austríaco em explorar o problema do conhecimento e de coordenação existente na sociedade, bem como um elemento a enriquecer sua crítica ao racionalismo construtivista, visto como uma abordagem que colocaria em xeque a própria humanidade em sua complexidade e extensão (fazendo, então, com que o institucionalismo hayekiano se encaixe na trajetória de pesquisa do autor). Na seção 3 , passa-se a fazer uma análise de conceitos institucionais hayekianos, explicando de modo sumário o que o austríaco entendia por ordem, lei, legislação e regras, e propondo uma divisão deste último conceito em regras de conduta, regras de conhecimento tácito, e regras mentais. Por fim, as considerações finais concluem o artigo. 


\section{O estudo das instituições no programa de pesquisa de Hayek}

A contribuição de Hayek à teoria institucional pode ser melhor compreendida a partir de uma perspectiva mais ampla da agenda de pesquisa subjacente a toda sua obra. Como esclarecem Garrison e Kirzner (1987), Boettke (1990, 2000, 2005) e O'Driscoll (1977), o entendimento de que a economia lida com o problema da coordenação trespassa a produção hayekiana, bem como a noção de que é através do funcionamento do processo de mercado que o sistema econômico dinamicamente caminha em direção a um maior grau de coordenação. ${ }^{l}$ Sob tal ângulo, pode-se entender a pesquisa institucional hayekiana como uma tentativa de aproximação desse problema, de quais são as instituições que melhor permitem a existência de maior grau de coordenação, no sentido de que o conhecimento difuso, subjetivo e tácito possa fluir de melhor maneira através dos participantes do processo de mercado, permitindo o aumento do grau de compatibilização dos planos individuais. Segundo Caldwell, "Hayek's central question is: What set of institutional arrangements might best assist fallible individuals to make better decisions and better use of their knowledge?" (Caldwell 2004, 286) e, de acordo com Boettke, "[d]espite the diverse nature of his research, it has a common theme: How do social institutions work, through the filter of the human mind, to coordinate human affairs?" (Boettke 1990, 62). Nas palavras do próprio Hayek:

$[T]$ he real problem is rather how it can be brought about that as much of the available knowledge as possible is used. This raises for a competitive society the question, not how we can "find" the people who know best, but rather what institutional arrangements are necessary in order that the unknown persons who have knowledge specially suited to a particular task are most likely to be attracted to that task (Hayek 1948b, 95).

Tendo estas perguntas condutoras da agenda de pesquisa de Hayek em mente, o objetivo da presente seção é tecer algumas considerações sobre o institucionalismo hayekiano. Em particular, interessa entender, em pri-

1 Não é objetivo deste trabalho adentrar no terreno do debate sobre a questão na unidade ou ruptura ao longo da vida intelectual produtiva de Hayek, que engloba mais de seis décadas. Para algumas das referências deste debate, ver, além dos textos já mencionados, os apêndices B e C de Caldwell (2004), bem como Fleetwood (1995, 1997), Soromenho (1994), Feijó (2000, cap. 4) e Boettke, Schaeffer e Snow (2010). 
meiro lugar, de que maneira o estudo das instituições emerge na agenda de pesquisa do austríaco e, em segundo lugar, como Hayek buscou esclarecer a relação entre, de um lado, regras, cultura e instituições e, de outro, o desempenho e sobrevivência de certo grupo social.

\subsection{As instituições como parte do desenvolvimento da agenda de pesquisa de Hayek}

Lewis (2014) argumenta que a preocupação de Hayek com o tema das instituições foi surgindo de maneira gradual com o desenvolvimento do projeto de pesquisa do austríaco. Na interpretação oferecida por aquele autor para o projeto hayekiano, os textos de Hayek, de 1937 e 1945, expõem o problema econômico como o problema do conhecimento, fazendo, assim, uma crítica epistemológica à teoria econômica.

Em uma palavra, no artigo de 1937, Hayek questiona a opção metodológica feita pelos economistas de estudar estados de equilíbrio - o que, em sua perspectiva, significa que os economistas pressupõem aquilo que deveriam explicar, ou seja, o equilíbrio. Para ele, é razoável imaginar que se possa alcançar equilíbrio individual, no sentido de que os planos de um indivíduo sejam coerentes entre si e com o conhecimento que ele possui. No entanto, a passagem do equilíbrio individual para o geral não é trivial, na medida em que o conhecimento é difuso e que o conhecimento considerado "dado" para uma pessoa não é "dado" para todos. Além disso, a ação esperada dos demais indivíduos é levada em consideração no momento de confecção dos planos de um agente específico. Contudo, nada garante que a ação esperada, expectativa essa formulada a partir de certa informação (parcial), seja aquela realmente levada a cabo pelos demais agentes. Com isso, criase um problema a respeito da (im)previsibilidade da ação e, portanto, da possibilidade de incompatibilidade de planos formulados pelos agentes, da existência de erros e da correção de expectativas.

Em tal contexto, o papel do cientista social é desvendar como o conhecimento pode ser mais bem distribuído, de modo a permitir a cada indivíduo prever melhor as ações das outras pessoas, resultando na possibilidade de formulação de planos individuais corretos. Isso, vale dizer, significa entender de que maneira o sistema econômico pode dinamicamente caminhar rumo a um maior grau compatibilização de planos e, assim, de coordenação. 
O que Hayek, especialmente em seu texto de 1945, destaca, é o papel do sistema de preços a atuar como uma espécie de sistema de comunicação da economia, através do qual o conhecimento difuso é sintetizado. Com isso, o sistema de preços torna-se capaz de atuar como um elemento central no aumento do grau de coordenação e de utilização do conhecimento total existente na economia.

Retornando à narrativa de Lewis (2014), o livro de psicologia de Hayek (1976b), publicado originalmente em 1952, aprofunda os fundamentos ontológicos àquela crítica epistemológica apresentada em 1937 e 1945. Finalmente, após 1960, seus trabalhos (e.g. Hayek 1960, 1973, 1976a, 1979,1988 ) procuram avançar na compreensão de como as instituições lidam com o problema do conhecimento, e, através de uma análise institucional comparativa, achar no liberalismo a maneira mais adequada de a sociedade tratar e conviver com ele. Em outras palavras, ao aprofundar-se no estudo das instituições, Hayek buscou explorar a maneira com que elas atuavam conjuntamente com o sistema de preços na explicação da transmissão de conhecimento e, assim, do aumento do grau de compatibilização de planos e da utilização do conhecimento total existente. ${ }^{2}$

$\mathrm{Na}$ abordagem hayekiana, como se sabe do debate do cálculo econômico socialista, a defesa do livre mercado em comparação ao planejamento socialista não está assentada principalmente na questão da diferença de incentivos existente entre os dois sistemas econômicos, mas sim no problema do conhecimento. É ao vincular sua pesquisa ao problema do conhecimento que Hayek pretende mostrar a impossibilidade do socialismo como mecanismo capaz de assegurar a distribuição da produção de acordo com algum suposto critério de justiça, bem como de se rearranjar a sociedade, planejando racionalmente a participação econômica individual, a composição do produto e sua distribuição.

Segundo Hayek, a defesa do socialismo está assentada, em primeiro lugar, na crença acrítica na capacidade racional humana característica do racionalismo construtivista ${ }^{3}$ e, em segundo lugar, e de forma relacionada, na

2 Para uma interpretação de que, em Hayek, outras instituições atuam de forma complementar ao sistema de preços, ver Vaughn (1999).

3 Hayek, por vezes, chama tal corrente de pensamento de construtivismo racionalista ("It seems to me that the best name for this kind of naïve rationalism is rationalist constructivism", Hayek, 1965, 85), por outras, de racionalismo construtivista ("a particular conception of the formation of social institutions, which I shall call 'constructivist rationalism' - a conception which assumes that all social institutions are, and ought to be, the product of deliberate design", Hayek 1973, 5). Ele chega a falar que "construtivístico" seria melhor que "construtivista": "Perhaps to show that we use the term 
falha de percepção sobre qual é o problema do conhecimento, ou seja, que o conhecimento presente de maneira difusa na sociedade sequer existiria fora do processo de mercado - ainda que existisse, tal conhecimento, por sua própria natureza subjetiva, não poderia ser coletado e organizado em uma espécie de comitê central de planejamento. Ademais, o socialismo não conseguiria distinguir entre regras planejadas e criadas intencionalmente pelo homem daquelas que também são criadas pela ação humana, porém como consequência não intencional dela. Daí a confusão que leva à crença de que é possível racionalmente reconstruir o padrão moral e as regras que caracterizam determinada sociedade.

O que Hayek argumenta é que as instituições de origem espontânea e selecionadas de forma evolucionária, mesmo que geralmente não sejam seguidas de boa vontade e que, a princípio, não demonstrem ter influência positiva sobre o grupo que as executa, podem sim ser importantes para seu sucesso; o fracasso do socialismo, pensa o austríaco, reside no desejo que esta doutrina representa de refundar a sociedade e suas práticas segundo um critério explicitamente racional, em particular o funcionamento de seu sistema econômico. Isso a leva a descartar as práticas tradicionais que são importantes no processo de crescimento e utilização do conhecimento social total (que é difuso, tácito e subjetivo) em favor da organização supostamente racional.

O interesse no estudo da evolução das instituições, assim, ganha especial relevância, na medida em que ajude no entendimento de como se dá esse processo tão importante para o progresso da humanidade, apesar de pouco apreciado - e muitas vezes criticado como conservadorismo, ${ }^{4}$ e também

in a critical sense, 'constructivistic' is better than 'constructivist'” (Hayek 1970, 3, n. 2) e "since 'constructivist' appears to some people still to carry the commendatory connotation derived from the adjective 'constructive', I felt it advisable, in order clearly to bring out the deprecatory sense in which I use that term (...) to employ instead the, I am afraid, still more ugly term 'constructivistic'" (Hayek 1982, xix).

4 Hayek reservou o posfácio (chamado Why I am not a conservative) a seu livro de 1960 à explicação da diferença central entre liberalismo e conservadorismo. Em uma palavra, seu argumento é que o conservador repudia mudanças e transformações, em particular das instituições e das regras morais, ao passo que o liberal é, a princípio, simpático às mudanças, desde que elas aconteçam através do processo competitivo espontâneo e evolucionário. Ver também Hayek (1970): "The proper conclusion from the considerations I have advanced is by no means that we may confidently accept all the old and traditional values. Nor even that there are any values or moral principles, which science may not occasionally question. (...) The consequence of what I have said is merely that we can never at one and the same time question all its values. Such absolute doubt could lead only to the destruction of our civilisation and - in view of the numbers to which economic progress has allowed the human race to grow - to extreme misery and starvation". (Hayek 1970, 19) 
porque seu produto (regras de comportamento tradicionais, por exemplo) está em constante pressão, por um lado, pelo comportamento instintivo e, por outro, pelo comportamento supostamente guiado racionalmente.

No ponto de vista hayekiano, é através do mecanismo de mercado - e, por tabela, do comportamento associado a ele - que as pessoas, ao seguirem regras espontâneas, conseguem, numa ordem complexa, atingir por meio de suas ações uma gama enorme de outros indivíduos. Ao mesmo tempo, é essa a forma de se beneficiarem do conhecimento e ações também de pessoas que não são suas conhecidas. Nessa perspectiva, a ordem ampliada só se torna possível se a sociedade se dispõe, ainda que inconscientemente, a tirar proveito dessa vasta rede de cooperação anônima necessária ao aumento do grau de divisão do trabalho e do conhecimento e à utilização, por cada um, do trabalho e do conhecimento alheios. É o seguimento compartilhado de regras - inclusive regras de percepção, como as que se ligam ao sistema de preços - que promove o erguimento e manutenção das ordens sociais que nos permitem tanto "to do things by circumstances of which we are largely unaware and which produce results that we do not intend" (Hayek 1988, 14) quanto "[to] serve people whom we do not know, and even of whose existence we are ignorant" (Hayek 1988, 14).

Assim, Hayek assenta a existência da grande sociedade nas regras tradicionais e nas instituições espontâneas e evolucionárias, de seguimento muitas vezes inconsciente, de maneira que, ao seguir os sinais abstratos e escolher cooperar e atender as necessidades de quem esteja além de sua percepção, o indivíduo consegue beneficiar um número muito maior de pessoas e se aproveitar do conhecimento difuso, subjetivo e tácito existente numa sociedade complexa.

\subsection{A narrativa de Hayek para a importância social das regras e instituições}

De acordo com Hayek (1973, cap. 1; 1979, cap. 11; 1988, cap. 1), o comportamento individual é resultado da ação conjunta de três fatores: os instintos, a razão e as regras de conduta (que ficam entre as duas categorias anteriores), ${ }^{5}$ sendo que tais regras são mediadas pelo lugar, época, história

5 "Our innate instincts are here in conflict with the rules of reason we have learned, a conflict we can resolve only by limiting coercion to what is required by abstract rules and by abstaining from enforcing what can be justified only by the desire for particular results" (Hayek 1979, 150)."[C]ustom and tradi- 
e experiência individuais e particulares. ${ }^{6}$ Segundo aponta o austríaco, os instintos, que não deixam de ser tipos de regras (Hayek 1967a), são impulsos internos referentes à percepção e à ação hereditários que expressam uma evolução de milhões de anos que ajudou a constituir o ser humano no sentido biológico do termo, inclusive (Hayek 1988, cap. 1). Eles eram importantes mecanismos que garantiam a sobrevivência dos pequenos grupos tribais, na medida em que produziam nos membros de cada grupo objetivos concretos e comuns a serem perseguidos, bem como percepções sobre riscos e oportunidades que poderiam ser exploradas. Ou seja, a atuação dos instintos geneticamente herdados levava os indivíduos a cooperarem para a consecução de objetivos comuns, como a busca por alimento, abrigo e segurança. Eram eles o mecanismo através do qual os pequenos grupos, compostos por membros que se conheciam e eram atados por laços de solidariedade, podiam ser coordenados - através da existência de objetivos comuns gerados pela percepção também socialmente compartilhada de oportunidades e perigos. Entre os instintos humanos Hayek destaca o da solidariedade e do altruismo, "instincts applying to the members of one's own group but not to the others" (Hayek 1988, 12).

Contudo, a coordenação e cooperação não anônima baseada nos instintos e nos objetivos e percepções compartilhadas não podem ser levadas muito adiante como mecanismo de coesão social, na perspectiva de Hayek. ${ }^{7}$ Tais instrumentos podem ser úteis e importantes para pequenos grupos - "small roving bands or troops” (Hayek 1988, 11), nas palavras do austríaco - e continuar a influenciar o comportamento individual na Great society, mas são incapazes de, sozinhas, exercerem o mesmo papel virtuoso em um

tion stand between instinct and reason - logically, psychologically, temporally" (Hayek 1988, 23, itálico no original). "I want to call attention to what does indeed lie between instinct and reason, and which on that account is often overlooked just because it is assumed that there is nothing between the two. That is, I am chiefly concerned with cultural and moral evolution, evolution of the extended order, which is, on the one hand (...) beyond instinct and often opposed to it, and which is, on the other hand (...), incapable of being created or designed by reason" (Hayek 1988, 21, itálico no original).

6 "The rules upon which different individual members of a group will at any moment act may therefore be different either because the drives or external circumstances acting upon them make different rules applicable, or because different individuals according to age, sex, status, or some particular state in which each individual finds itself at the moment" (Hayek 1967a, 68).

7 "[O]nly in the small groups of primitive society can collaboration between the members rest largely on the circumstance that at any one moment they will know more or less the same particular circumstances. Some wise men may be better at interpreting the immediately perceived circumstances or at remembering things in remote places unknown to the others. But the concrete events which the individuals encounter in their daily pursuits will be very much the same for all, and they will act together because the events they know and the objectives at which they aim are more or less the same. The situation is wholly different in the Great or Open Society where millions of men interact and where civilization as we know it has developed" (Hayek 1973, 14). 
grupo social maior e mais complexo. Em sua visão, com o crescimento do grupo social rumo a se tornar uma ordem mais complexa, ficaria cada vez mais difícil a existência tanto de objetivos comuns quanto de percepções compartilhadas e de métodos julgados como apropriados ao alcance daqueles fins. Com isso, basear a sociabilidade apenas nos instintos primários se torna progressivamente inviável. De acordo com ele, "[c]ooperation, like solidarity, presupposes a large measure of agreement on ends as well as on methods employed in their pursuit" (Hayek 1988, 19).

É neste ponto que Hayek traz à tona a necessidade da existência de regras de comportamento socialmente compartilhadas como condição necessária à existência de uma ordem espontânea ampla. Nesse sentido o austríaco se coloca, em sua própria interpretação, na mesma linha de pesquisa dos fundadores da ciência econômica, que se preocupavam em como poderia acontecer de se chegar a uma ordem social tão larga que excederia a capacidade de criação e organização da razão humana. ${ }^{8}$

Se os instintos foram importantes para o processo milenar de formação da própria espécie humana, é a existência de limites a esses mesmos instintos que liberta algumas das amarras ao crescimento do grupo social, na medida em que não se exige mais o compartilhamento de percepções e objetivos como uma condição à existência de cooperação (agora, anônima) social.

A relação entre instintos e regras é complexa ao invés de unidirecional. $\mathrm{Na}$ perspectiva hayekiana, as regras de conduta podem ser classificadas como restrições culturais ao comportamento instintivo biologicamente herdado, de modo que regras e instintos estão em recorrente oposição e conflito. ${ }^{9}$ As regras são construções coletivas não intencionais, explícitas ou implícitas, transmitidas por meio da cultura que provocam uma espécie de inclinação à rejeição ou desgosto por certos tipos de impulsos internos instintivos. As regras, ainda, exercem uma função coordenadora dos grupos sociais amplos. De um ponto de vista hayekiano, a adesão a regras é importante para a expansão do grupo social ao "libertar" os indivíduos de seguirem seus instintos de percepção e objetivos comuns. Daí que Hayek (e. g. 1979, cap. 11; 1988, cap. 1), por vezes, dá mais ênfase ao papel das

8 De acordo com Hayek, já nos fundadores da economia pode ser encontrada uma explicação de cunho evolucionário para esse tipo de investigação. Em suas palavras, "Economics has from its origins been concerned with how an extended order of human interaction comes into existence through a process of variation, winnowing and sifting far surpassing our vision or our capacity to design" (Hayek 1988, 14).

9 Hayek afirma que mesmo entre os animais as regras se opõem aos instintos. Em suas palavras, "[t]he conflict which will often arise between immediate desires and the built-in rules or inhibitions is well attested by the observation of animals" (Hayek 1973, 76). 
regras (de conduta) como restrições ao comportamento benevolente do que como uma espécie de controle do egoísmo exacerbado e da "guerra de todos contra todos". 10

Em Hayek há ao menos três maneiras correlacionadas pelas quais as práticas selecionadas evolucionariamente expressam sua colaboração para o sucesso de um grupo social: o crescimento (no sentido demográfico), a utilização do conhecimento disperso e a manutenção da ordem (Hayek 1988, introdução).

Como já dito, a abordagem institucional de Hayek surge como resposta ao problema mais geral com que o austríaco se depara no seu esforço de compreensão da sociedade descentralizada e de cooperação anônima, qual seja, o problema do conhecimento. O conjunto de práticas selecionado pelo processo evolucionário e, portanto, espraiado no tempo e no espaço, é precisamente aquele que caracteriza uma ordem em que há uma melhor articulação entre os fragmentos do conhecimento que se encontra difuso na sociedade. Hayek encontra no arcabouço institucional-jurídico típico de uma sociedade livre - ou seja, em que as regras possuem caráter negativo, em que prevaleça a rule of law e em que as pessoas estejam livres do arbítrio alheio - a possibilidade de promoção dessa articulação.

Nesse tipo de ambiente, os indivíduos desfrutam da liberdade de utilizarem seu conhecimento local e muitas vezes inarticulado em seu próprio favor, e as instituições existentes numa sociedade coordenada através do livre mercado promovem a conversão desse interesse privado em benefícios alheios; tem-se, então, a cooperação anônima não intencional. Dessa forma, Hayek (1988, introdução) relaciona o comportamento de acordo com práticas selecionadas através da evolução como uma condição para a existência da "extended order" (Hayek 1988, 6), quando fala, por exemplo, sobre "the traditions that formed the extended order that made civilization possible" (Hayek 1988, 7). Dois possíveis motivos podem ser elencados para isso. Em primeiro lugar, de forma coadjuvante, a razão mais atrelada ao dilema hobbesiano; nesse caso, a internalização de certo padrão moral tradicional evitaria tanto a guerra de todos contra todos quanto a tirania do soberano. Em segundo lugar, mais de acordo com a obra mais ampla de Hayek, é a possibilidade de utilização do conhecimento existente em suas diferentes dimensões na sociedade trazida pela evolução de tais práticas. Do ponto de vista de Hayek, é possível que uma pequena comunidade con-

${ }^{10}$ Aliás, para ele, "[t]he primitive individualism described by Thomas Hobbes is hence a myth. The savage is not solitary, and his instinct is collectivist" (Hayek 1988, 12). 
siga viver com um comando central e baseada na cooperação não anônima, e com as motivações altruístas; contudo, com o crescimento deste grupo tal forma de sobrevivência acabaria por destruir a ordem.

Assim, a extended order deve estar assentada sobre a cooperação anônima e a utilização das práticas de origem espontânea e que são selecionadas exatamente por oferecerem ao grupo a possibilidade de aumentar sua eficiência perante outros através do crescimento do conhecimento e do grau de sua utilização. Nesse caso, a ordem tem origem espontânea, sem prévio planejamento, e, por isso mesmo, pode alcançar um grau de complexidade muito maior do que aquela que tivesse sido planejada ex-ante, racionalmente, já que a capacidade humana de previsão de todas as consequências que certa ação gerará é bastante limitada para lidar com a complexidade dos fenômenos inerentes aos grandes grupos. Para Hayek, reconhecer tal limite do uso da racionalidade é uma virtude; o desejo de se redesenhar racionalmente a sociedade e suas regras, característica inúmeras vezes associada por Hayek à linha de pensamento do "racionalismo construtivista", ao contrário, acaba por cair no erro de se aderir a uma "naive and uncritical theory of rationality, an obsolete and unscientific methodology" (Hayek $1988,8) \cdot{ }^{11}$

De acordo com Hayek, o processo de evolução atua de modo a selecionar o grupo que adota as regras que melhor permitam a ele se adaptar ao ambiente constantemente sujeito a mudanças. A manifestação do sucesso ou fracasso do grupo se dá pelo crescimento tanto econômico quanto demográfico relativamente a outro grupo que siga um conjunto de regras e práticas distinto. Assim, o papel da evolução é permitir que o conjunto de regras que melhor permita a um grupo social prosperar e crescer numericamente prevaleça.

\section{Sumário analítico de alguns conceitos institucionais hayekianos}

Feita uma discussão sobre alguns aspectos do institucionalismo de Hayek e sua relação com o liberalismo defendido pelo austríaco, cumpre esclarecer o entendimento do autor para alguns conceitos utilizados.

${ }^{11}$ Por oposição ao racionalismo construtivista, Hayek se identificava com a linha do racionalismo crítico. Ver Hayek (1965). 


\subsection{Regras e cultura}

Um conceito central na economia institucional de Hayek é o de regras que servem como uma espécie de caracterização de certa ordem. Denominações alternativas que possuem significado semelhante (Hayek 1988, introdução e cap.1; 1973, cap. 4), ao menos em uma dimensão do termo, são práticas, já que o seguimento de regras é um elemento que atua no sentido de produzir regularidade no comportamento individual, ou mesmo moralidade tradicional. Hayek (1988, cap. 1) faz a distinção entre dois usos para o termo moral: o primeiro é o referente a uma espécie de moralidade natural e o segundo dizendo respeito à moralidade cultural. É a esse segundo sentido que o austríaco faz referência quando utiliza o termo moral.

As regras de origem espontânea possuem um caráter social e coordenador na medida em que Hayek fala delas usualmente relacionando-as, por exemplo, com a criação e utilização do conhecimento social inarticulado e com a distribuição do produto econômico (e.g. Hayek 1988, introdução). Elas também são, de certa forma, a manifestação do conhecimento acumulado pelo grupo social. As regras que fazem parte da cultura de um povo são ao mesmo tempo resultado dos próprios limites da racionalidade dos agentes (e não sua origem) e mecanismo para: (i) utilizar-se o conhecimento social acumulado por gerações e (ii) dar racionalidade não necessariamente à ação individual, mas sim ao ambiente e às regras. Regras compõem a cultura de certa sociedade. Elas atuam como instrumento de construção não intencional e manutenção de ordens auto-organizadas e complexas, já que a capacidade racional de construção e entendimento de todas suas facetas, qualidades e manifestações particulares é limitada.

As regras de que trata Hayek, por serem parte da cultura de um povo, devem ter sua forma de transmissão caracterizada de modo distinto da herança genética, podendo ocorrer por "tradition, teaching and imitation" (Hayek 1988, 12); a cultura influencia o comportamento individual, mas de modo distinto da herança genética, que por sua vez atua através dos instintos.

Para Hayek (1973, caps. 1 e 4), cultura "consists of a complex of practices or rules of conduct” (Hayek 1973, 17). Ela é transmitida de geração a geração (não necessariamente de pai para filho) e representa a solução que o grupo encontrou, geralmente sem saber o motivo exato, para os pro- 
blemas enfrentados e que por isso acabaram levando à sua sobrevivência. Nesse sentido, não é propriamente o conjunto de regras ou a cultura que são selecionados; a seleção é do grupo que tenha aderido a tal cultura, ou melhor ainda, da ordem social que é a estrutura supraindividual formada pela regularidade do seguimento de regras de comportamento.

Para Hayek (e. g. 1967a; 1968) regra também descreve a forma pela qual determinada regularidade no comportamento individual, seja ela deliberada e voluntária ou não, pode ser descrita e permitir a previsão sobre o comportamento alheio: "a rule may effectively govern action in the sense that from knowing it we can predict how people will act, without it being known as a verbal formula to the actors" (Hayek 1968, 81).

Segundo o austríaco, convenção é uma regra de comportamento a que o indivíduo se submete quando participa de um grupo social, sem necessidade de coerção, ${ }^{12}$ só rompendo quando consegue enxergar justificativas plausíveis para tanto. ${ }^{13}$ Convenção não pode ser justificada em termos racionais, em geral, chegando muitas vezes a parecer absurda. Ela é fruto do processo evolucionário não teleológico e torna o comportamento de outrem previsível. ${ }^{14}$

Em Hayek podemos encontrar ao menos três tipos correlacionados, mas distintos, de regras: as regras de conhecimento tácito, as regras de conduta e as regras mentais. ${ }^{15}$ Essa classificação é explicada a seguir.

12 "[T] raditions and conventions (...) evolve (...) without being enforceable, [and] establish flexible but normally observed rules that make the behavior of other people predictable in a high degree" (Hayek 1946, 23). "[C]oercion can probably be kept to a minimum in a society where conventions and tradition have made the behavior of man to a large extent predictable" (Hayek 1946, 24).

13 Segundo Hayek, a adesão às regras convencionais se dá "not merely so long as one understands the reason for them but so long as one has no definite reasons to the contrary" (Hayek 1946, 23).

14 "[T] he individual, in participating in the social process, must be ready and willing to adjust himself to changes to submit to conventions which are not the result of intelligent design, whose justification in the particular instance may not be recognizable, and which to him will often appear unintelligible and irrational" (Hayek 1946, 22).

${ }^{15}$ Note-se a existência de um paralelo entre a classificação ora proposta de três tipos de regras em Hayek (i. regras de conhecimento tácito, ii. regras de conduta e iii. regras mentais), com alguns dos papéis que as instituições desempenham na influência sobre o comportamento e o pensamento dos indivíduos segundo a classificação de Dequech (e.g. 2011): i. papel cognitivo prático, ii. papel restritivo e iii. papel cognitivo informacional e fundamental. 


\subsubsection{Regras de conduta}

Ao tratar de regras, por vezes Hayek diz respeito às regras de conduta que têm um caráter negativo, restritivo, a atuar sobre o comportamento humano. Aqui, o termo moral se adequa bem. Elas delimitam o espaço, de forma consciente ou não, no qual o comportamento instintivo individual pode se manifestar. Nas palavras do austríaco, "rules of conduct will always act only as a restraint on actions induced by other causes" (Hayek 1967a, 69). Nesse sentido, regra é o oposto de ordem (comando), já que a última impõe de maneira positiva os caminhos que devem ser traçados pelo indivíduo, ao passo que a primeira se limita a indicar, na forma de princípios abstratos, ${ }^{16}$ que o indivíduo tem responsabilidade pelos seus atos, de modo que ele possa adaptar suas ações a elas.

Hayek (1973, cap. 4) trata da existência de um tipo modificado de regras de conduta que ele chama de regras normativas, quais sejam, aquelas que descrevem como deveria acontecer uma sequência de ações, ao invés de meramente descreverem tal sequência de eventos e atitudes. Ele chama de regras normativas aquelas que surgiram como regras de conduta implícitas, não verbalizadas, mas que, com o passar do tempo, como que emergiram e se tornaram aos poucos explícitas, sujeitas à adesão consciente e passíveis de contestação. ${ }^{17}$ Essa possibilidade de falta de adesão produz muitas vezes a existência de uma pressão externa, uma imposição explícita a que tal regra seja seguida, ainda que não se encontre motivo racional ou explicação para isso.

$\mathrm{Na}$ abordagem hayekiana, as práticas comportamentais tradicionais são herdadas culturalmente, ou seja, elas são uma parte do que Hayek chama de instituições de origem espontânea que surgem concomitantemente à emergência não só da civilização, mas da própria razão humana. Por isso, tentar encontrar um fundamento racional para as regras morais é um esforço, em certo sentido, anacrônico (Hayek 1979, Epílogo). Elas passam por um processo evolucionário e são seguidas geralmente de forma inconsciente, sem que haja necessariamente, para elas, justificativa racional ou motivo claro para que sejam cumpridas.

16 " $[\mathrm{A}] \mathrm{n}$ individualistic order must rest on the enforcement of abstract principles rather than on the enforcement of specific orders" (Hayek 1946, 19).

17 "It seems that the specific character usually ascribed to 'norms' which makes them belong to a different realm of discourse from statements of facts, belongs only to articulated rules, and even there only once the question is raised as to whether we ought to obey them or not" (Hayek 1973, 79). 
Nesse sentido, pode-se pensar nessas práticas como uma espécie de código moral. Segundo Hayek:

They will often merely determine or limit the range of possibilities within which the choice is made consciously. By eliminating certain kinds of action altogether and providing certain routine ways of achieving the object, they merely restrict the alternatives on which a conscious choice is required. The moral rules, for example, which have become part of a man's nature will mean that certain conceivable choices will not appear at all among the possibilities between which he chooses (Hayek 1962, 56)

Contudo, não é tão claro se tais práticas morais de origem espontânea e frutos de seleção evolucionária devem ser acompanhadas de algum tipo de pressão externa ou se é admitida a possibilidade de serem internalizadas. O que Hayek admite é uma relação inversa entre internalização das regras de comportamento e necessidade de coerção: "freedom has never worked without deeply ingrained moral beliefs and (...) coercion can be reduced to a minimum only where individuals can be expected as a rule to conform voluntarily to certain principles" (Hayek 1960, 62).

O austríaco admite a possibilidade de elas serem implícitas ou explícitas: "although learnt, morals do not necessarily always operate as explicit rules" (Hayek 1988, 13). Contudo, isso não é o mesmo que afirmar a chance de serem internalizadas ou não. É possível, por exemplo, que uma regra seja implícita, mas não seja internalizada, ou que seja explícita mas internalizada. Tais padrões de comportamento não necessariamente são racionais, no sentido de que nem sempre são seguidos após cálculos utilitaristas de ganhos ou perdas advindas da adesão a tais regras, tanto pelo comportamento originado pela regra quanto pelas consequências, diante da sociedade, da (não) adesão às regras. As pessoas os seguem, em geral, simplesmente porque, por hábito, sempre fizeram isso: "though we do not know their significance and may not even be consciously aware of their existence" (Hayek 1960, 62). Tanto que Hayek os classifica como hábitos e tradições firmemente estabelecidos: 
$[M]$ ost of the time, members of our civilization conform to unconscious patterns of conduct, show a regularity in their actions that is not the result of commands or coercion, often not even of any conscious adherence to known rules, but of firmly established habits and traditions (Hayek 1960, 62).

Nesse sentido, as regras de conduta possuem um caráter tácito.

\subsubsection{Regras de conhecimento tácito}

Em boa parte das vezes em que trata de regras, Hayek as relaciona ao conhecimento tácito, inarticulado, possuído pelos indivíduos. Como se sabe, Hayek, por diversas vezes ao longo de sua obra, deu destaque ao caráter tácito do conhecimento, o knowing how, em contraposição ao conhecimento claro, verbalizado e passível de formulação, o knowing that (Andrade 2004). O conhecimento tácito de que trata Hayek diz respeito à maneira de agir diante de determinada situação a partir da adesão, ainda que de maneira inconsciente, a certas regras, maneira esta que o indivíduo é incapaz de formular, justificar e transmitir fácil e perfeitamente. Segundo Hayek:

[T] he ability of small children to use language in accordance with the rules of grammar and idiom of which they are wholly unaware (...). The phenomenon is a very comprehensive one and includes all that we call skills. The skill of a craftsman or athlete which in English is described as 'knowledge how' (to carve, to ride a bycicle, to ski, or to tie a knot) belongs to this category. It is characteristic of these skills that we are usually not able to state explicitly (discursively) the manner of action which is involved. (Hayek 1962, 43)

Ao tratar de regras de conhecimento tácito, deve-se ter em mente especialmente o processo de imitação de que fala Hayek (1962). Isso diz respeito a, por exemplo, maneiras específicas de movimentar o corpo, de fazer gestos, e mesmo de falar. Pode-se pensar também que determinados tipos de 
trabalho são caracterizados pelo conhecimento tácito, em que a pessoa sabe "como fazer", mas não consegue verbalizar e tampouco ensinar facilmente o serviço a algum aprendiz. Assim, "the 'know how' consists in the capacity to act according to rules which we may be able to discover but which we need not be able to state in order to obey them" (Hayek 1962, 44).

A distinção das regras de conhecimento tácito em relação às regras de conduta diz respeito a que estas últimas exercem uma influência negativa sobre o comportamento, ao ditar o que o sujeito não deve fazer. Por seu turno, as regras de conhecimento tácito podem ser compreendidas como apresentando uma ação positiva sobre o comportamento, já que não possuem propriamente o caráter de delimitar a ação, mas de determinar a ação. Elas não dizem quais gestos a pessoa não deve fazer, quais movimentos a pessoa não deve ter em seu trabalho, etc., mas dizem quais gestos são adequados para tais e tais situações, como o trabalho deve ser executado - ainda que o indivíduo não tenha consciência de que age de acordo com regras, não consiga verbalizá-las ou transmiti-las facilmente, ou sequer justificar a razão de agir conforme tais regras.

Nas palavras de Hayek:

Whether is the bird which is induced to fly (or preen, scratch, shake itself, etc.) by the sight of other birds doing so, or man induced to yawn or stretch by seeing others doing the same, or the more deliberate imitation practised in mimicry or learning a skill, what happens in all these instances is that an observed movement is directly translated into the corresponding action, often without the observing and imitating individual being aware of the elements of which the action consists or (in the case of man) being able to state what he observes and does. Our capacity to imitate someone's gait, postures, or grimaces certainly does not depend on our capacity to describe these in words. (Hayek 1962, 47-48) ${ }^{18}$

${ }^{18}$ Como se depreende deste trecho, as regras de conhecimento tácito estão intimamente vinculadas à percepção dos gestos, ações, formas de agir, etc., dos outros, ou seja, dependem da percepção das regras que governam as ações das outras pessoas - percepção esta que, na presente classificação, fica melhor enquadrada nas regras mentais. 
O conceito hayekiano de regras, especialmente o de regras de conhecimento tácito, é semelhante ao de heurística, uma forma determinada de se resolver problemas transmitido ao longo do tempo.

\subsubsection{Regras mentais}

Em Hayek, pode-se também encontrar uma dimensão mental das regras. ${ }^{19}$ Para o austríaco, as regras mentais são herdadas culturalmente e servem como uma espécie de critério de classificação e organização das percepções caóticas que nos atingem a todo o momento.

$\mathrm{Na}$ dimensão mental as regras levam a uma organização e permanente rearranjo dos constantes estímulos sensoriais que nos bombardeiam de modo aparentemente caótico a todo instante. Essa organização se dá segundo alguns critérios que são aderentes às próprias regras. Esse infindável processo de rearranjo, classificação e diferenciação organiza os estímulos sensoriais, permitindo à mente, que na perspectiva hayekiana é considerada uma estrutura abstrata de regras de classificação, ordenar os fenômenos e objetos ao nosso redor de acordo com os critérios ditados pelas regras mentais, de modo a associar os estímulos sensoriais a certas características do mundo que conhecemos, capacitando-nos a agir nele.

O aparente caos característico dos estímulos sensoriais que nos alcançam continuamente é reflexo da complexidade do mundo que nos rodeia. Nesse sentido, as regras podem ser compreendidas como uma solução para o dilema entre nossa capacidade cognitiva limitada e o ambiente complexo em que estamos inseridos. A racionalidade limitada dos indivíduos diante da complexidade do ambiente, portanto, ajuda a entender porque, para Hayek, as regras espontâneas evolucionárias são tão importantes para a sobrevivência da sociedade na dimensão que conhecemos hoje.

Hayek trata da mente humana como um sistema de interpretação do mundo através do conhecimento produzido no passado seja pelo próprio indivíduo, seja pela espécie (Di Iorio 2010). As percepções que temos são reflexos imperfeitos e incompletos da realidade exterior, baseados na

${ }^{19}$ Neste artigo a percepção guiada por regra de que fala Hayek (1962) se enquadra na categoria de regras mentais: "[r]ules which we cannot state thus do not govern only our actions. They also govern our perceptions, and particularly our perceptions of other people's actions." (Hayek 1962, 45) 
experiência pretérita. A relação entre estímulos sensoriais e percepção mental é bastante imperfeita. De acordo com Di Iorio,

It follows that sensory qualities, that is, the blue of the sky or the green of the grass, cannot be regarded as objective and ultimate properties of reality, but as mental constructions. Hayek maintains that the mind reproduces only conjecturally, partially and approximately the external world. (Di Iorio 2010, 181-182)

A estrutura de regras classificativas que é a mente capta alguns elementos abstratos dos estímulos, identifica alguns traços deles e encontra seu lugar de acordo com o conhecimento pretérito que possuímos. O reconhecimento de certas qualidades abstratas dos objetos e fenômenos que chegam a nós e que permitem sua identificação, organização e classificação por analogia, acontece por meio de critérios oriundos de nosso passado individual ou grupal (Hayek 1976b).

Além disso, na proposta de Hayek, um tipo de situação provoca certa disposição no indivíduo que conduz a um padrão de resposta. Tal resposta ao estímulo não é imediata, mas o cérebro como que identifica características gerais do estímulo e produz certas disposições para o comportamento. Isto remete à "primazia do abstrato" de que fala Hayek (1969). Abstração é o que constitui a mente, a adaptação à ignorância de fatos particulares. A mente não pode lidar plenamente com a complexidade, então seleciona de modo não deliberado características gerais da realidade recebidas através dos estímulos sensoriais e, a partir de diferentes disposições e padrões de resposta (oriundos de regras) e das características abstratas dos estímulos, gera padrões de comportamento específicos (Hayek 1973, cap. 1). ${ }^{20}$

${ }^{20}$ Hayek faz duas observações com o intuito de esclarecer o papel das regras aqui chamadas de mentais. Em primeiro lugar, agir a partir de regras que não são por nós conhecidas não significa dizer que o comportamento seja instintivo ou intuitivo. Em segundo lugar, a admissão de regras não conscientes não implica a aceitação da existência de uma espécie de subconsciente autônomo racional: " $[\mathrm{t}]$ here are two possible misconceptions of the position taken which we must try to prevent. One derives from the fact that action which is guided by rules we are not aware of is often described as 'instinctive' or 'intuitive'. There is not much harm in these words except that both, and specially 'intuitive', usually refer to the perception of the particular and relatively concrete, while what we are here concerned with are capacities determining very general or abstract properties of the actions taken. As commonly used, the term 'intuitive' suggests an attribute not possessed by abstract rules which we follow in our actions, and for this reason it had better be avoided. The other possible misunderstanding of our position is the impression that the emphasis we place on the non-conscious character of many of the rules which govern our action is connected with the conception of an 
Duas marcas desse processo de classificação devem ser ressaltadas: a primeira, como explicado acima, é que, para Hayek, a correspondência entre realidade objetiva e nossa percepção é imperfeita, ${ }^{21}$ e a segunda é que esse tipo de processo de ordenamento dos estímulos sensoriais segundo regras é uma das facetas que assume o conhecimento tácito que Hayek procura destacar em diversos pontos de sua obra, conhecimento inarticulado, não passivo de verbalização e incapaz de ser recolhido e processado por uma única mente ou comitê central.

A Tabela 1 abaixo resume a discussão acerca da classificação proposta para os diferentes tipos de regras e os instintos em Hayek:

Tabela 1 - Características de instintos e de regras em Hayek

\begin{tabular}{|c|c|c|c|c|}
\hline & Instintos & $\begin{array}{c}\text { Regras de } \\
\text { Conhecimento Tácito }\end{array}$ & Regras de Conduta & Regras Mentais \\
\hline $\begin{array}{c}\text { Transmissão e } \\
\text { Compartilhamento }\end{array}$ & Biológica e individual & Cultural & Cultural & Cultural \\
\hline $\begin{array}{c}\text { Atuação Usual } \\
\text { Sobre Indivíduo }\end{array}$ & $\begin{array}{c}\text { Positiva sobre } \\
\text { Comportamento }\end{array}$ & $\begin{array}{c}\text { Positiva sobre } \\
\text { Comportamento }\end{array}$ & $\begin{array}{c}\text { Negativa sobre } \\
\text { Comportamento }\end{array}$ & $\begin{array}{c}\text { Organização, } \\
\text { Interpretação e } \\
\text { Resposta a Estímulos } \\
\text { Sensoriais }\end{array}$ \\
\hline
\end{tabular}

\subsection{Ordem}

Outro conceito central à economia institucional de Hayek é o de ordem. ${ }^{22}$ Pode-se compreender que, na perspectiva do austríaco, ordem é uma espécie de padrão ou estrutura supraindividual formada como consequência

unconscious or subconscious mind underlying the theories of psychoanalysis or 'depth-psychology'. But although to some extent the two views may aim at an explanation of the same phenomena, they are in fact wholly different (...). We shall not use, and in fact regard as unwarranted and false, the whole conception of an unconscious mind which differs from the conscious mind only by being unconscious, but in all other respects operates in the same, rational, goal-seeking manner as the conscious mind. Nothing is gained by postulating such a mystical entity." (Hayek 1973, 31)

${ }^{21}$ Segundo Di Iorio (2010), em texto sobre Hayek, o avanço do conhecimento científico serve como mecanismo para correção deste tipo de desvio. Em suas palavras, "[n]atural sciences tend to "revise" and "correct" our sensorial categorizations in order to grasp the features of the physical world" (Di Iorio 2010, 183). Ver também Hayek (1976b).

22 'There is no adequate term other than 'order' by which we can describe it, although 'system', 'structure' or 'pattern' may occasionally serve instead". (Hayek 1973, 35). Adiante, o austríaco explica que "[i]n recent discussion the term 'system' is often used in much the same sense in which I use here 'order', which still seems to me preferable". (Hayek 1973, 155, n. 1). No entanto, ao rever sua trilogia dos anos 1970, Hayek reflete sobre o termo, e avisa seus leitores que "instead of 'order', in conformity with today's predominant usage, I occasionally now use 'system'”. (Hayek 1982, xix) 
não intencional do seguimento regular de regras por parte dos indivíduos. Estes, apesar de formarem a ordem através de suas ações, não planejavam que isso acontecesse, o que permite enquadrá-la no conjunto das consequências não intencionais da ação humana. Uma ordem emerge quando os indivíduos seguem regras que permitem a descoberta, utilização e transmissão de conhecimento entre eles. Tais qualidades das regras e das ordens correspondentes variam em grau, e Hayek argumenta que as instituições típicas do liberalismo são aquelas capazes de aumentá-las ao máximo, gerando um tipo especial de ordem, a ordem catalática (Lewis 2014).

Nas palavras de Hayek:

By 'order' we shall throughout describe a state of affairs in which a multiplicity of elements of various kinds are so related to each other that we may learn from our acquaintance with some spatial or temporal part of the whole to form correct expectations concerning the rest, or at least expectations which have a good chance of proving correct. (Hayek 1973, 36) ${ }^{23}$

O processo de seleção geralmente se dá, na perspectiva evolucionária de Hayek, no nível da ordem social e não no nível das regras propriamente dito. Dessa forma, é selecionada a ordem que carrega consigo certo conjunto de regras que a permita ser melhor adaptada que as demais, em particular ao reduzir a violência e estabelecer um ambiente pacífico dentro da ordem.

É possível se fazer dois tipos de corte para analisarmos as ordens. Em primeiro lugar, conforme visto acima, assim como em Hayek se encontra a admissão da existência de diferentes categorias de regras, o austríaco também fala da existência de dois tipos de ordem: uma mental e uma física. ${ }^{24}$ Além desta primeira divisão, Hayek (1973, cap. 2) também faz

${ }^{23} \mathrm{Na}$ dimensão física o termo ordem exprime a qualidade que as coisas possuem de serem estruturadas, apresentarem padrão que se propaga e que pode ser notada pela possibilidade de se fazer inferência acerca do comportamento e resposta das partes que formam tal ordem (Hayek 1973, 155, n. 3). Alguns anos depois, Hayek usa o termo ordem para a dimensão espacial (envolvendo, portanto, distintos indivíduos) e regularidade para a dimensão temporal da mesma espécie de fenômenos: "[r]egularity, derived from the Latin regula for rule, and order are of course simply the temporal and the spatial aspects of the same sort of relation between elements" (Hayek 1988, 15). No entanto, sobre alguns dos problemas com o livro de 1988, ver Caldwell (2004, 316-319), Boettke (2000, xxxiv, n. 21) e Ebenstein (2001, caps. 39 e 40).

${ }^{24}$ A rigor, Hayek (1976b), em sua psicologia teórica, trata de três tipos de ordens: a ordem sensorial, a ordem neural e a ordem física. A ordem física é objetiva e externa ao ser humano, e não pode ser 
a distinção entre a cosmos, que é um tipo de ordem que "forms of itself" (Hayek 1988, 20), ordem espontânea, potencialmente complexa, surgida sem um propósito definido e sem um planejamento anterior, grown (Hayek 1973 , 35), e a taxis, ordem artificial, planejada, simples (no sentido de não ser complexa), made (Hayek 1973, 35) e que objetiva o atendimento de determinado fim. A distinção feita por Hayek entre ordem espontânea e ordem artificial não deve ser confundida com a oposição natural versus antinatural. Como explica o austríaco (Hayek 1988, 19), a cosmos pode ser considerada tanto natural quanto antinatural. Ela é natural no sentido de ter emergido de maneira espontânea, independente do desígnio humano, através de um processo de seleção evolucionária, análoga à biológica, das regras que a cimentam. Por outro lado, ela é antinatural na medida em que não se baseia e nem favorece o comportamento instintivo (compreendido como natural) do ser humano, mas, ao contrário, vincula-se a regras que justamente limitam o espaço de ação desse tipo de comportamento.

De acordo com ele (Hayek 1967c), a falta de clareza usual, associada principalmente ao racionalismo construtivista, no que se refere à existência de uma terceira categoria - nem propriamente natural, tampouco precisamente artificial (no sentido de desenhado, planejado) - para as ordens que emergem como consequência não intencional da ação humana, é reflexo de uma imprecisão comum, qual seja, a falha que acontece quando se classifica todo tipo de fenômeno como sendo natural ou artificial, em particular quando se fala de instituições que surgem por causa da natureza (as physei) e das que surgem por planejamento (as thesei), ignorando a existência de uma categoria intermediária a lidar com fenômenos que são resultados não intencionais da ação humana.

Assim, cabe destacar que Hayek (e.g. 1967c) levanta três tipos de origem das ordens (ou das instituições, de forma mais geral): aquelas que são dadas pela natureza (physei), as que surgem pela ação humana e são fruto de planejamento (thesei) e, por fim, uma classe intermediária, aquelas que são consequências não intencionais da ação humana. O problema, para ele, é que boa parte do pensamento ocidental parece ignorar a existência desta

conhecida diretamente. Ela produz a ordem neural, que é formada pelas conexões neurais e que produz a ordem sensorial. Afirmar que existe uma ordem física objetiva não significa que elas estejam acessíveis ao cérebro humano. Ao contrário, a ordem neural é uma mera aproximação da ordem física, mas diferente dela, e a ordem sensorial é análoga à ordem neural. Butos e Koppl (2006) e Di Iorio (2010) apresentam explicações interessantes sobre a psicologia teórica de Hayek e sua relação com a teoria econômica. Curiosamente, em entrevista a James Buchanan em 1978, Hayek fala de seu livro de psicologia nos seguintes termos: "I still believe this is one of my more important contributions to knowledge." (Ebenstein 2001, 148) 
terceira categoria, tendendo a qualificar todas as ordens e/ou instituições ou como naturais, ou como planejadas. E, se foram resultado do planejamento racional humano, são passíveis de serem reconstruídas racionalmente, ou então eliminadas. Essa visão equivocada, na interpretação de Hayek, é resultado da ação conjunta de dois erros: em primeiro lugar, a disseminação de um tipo antropomórfico, quase animista, de visão da sociedade e, em segundo lugar, uma confiança exagerada na capacidade ilimitada da razão humana, a que ele chama de "presunção fatal" (Hayek 1988), e que são características de uma linha intelectual que ele repetidamente critica ao longo de seus textos: o racionalismo construtivista.

\subsection{Lei e legislação}

A distinção entre lei e legislação, do ponto de vista hayekiano, está atrelada à maneira como estas duas categorias surgem. Para Hayek (1973, cap. 4), lei é uma regra de conduta imposta aos indivíduos vivendo em determinada sociedade, sendo, portanto, uma norma. Ela é de origem espontânea ("has never been invented", Hayek 1973, 72), podendo inclusive ter surgido antes mesmo que a humanidade conscientemente passasse a formular esse tipo de regras, o que leva o austríaco a afirmar que "law is older than law-making” (Hayek 1973, 73). Não por acaso, de acordo com Hayek, o termo usado para se descrever os fenômenos regulares da natureza é o mesmo, lei. Isso porque, ele explica, a perspectiva humana era que tanto as regularidades apresentadas no mundo natural quanto as regras que governam a conduta humana em sociedade não podiam ser atribuídas ao desígnio humano, no sentido de que não eram fruto do desejo, planejamento ou ação direta de um homem, mas eram como que externas à humanidade, frutos seja da divindade, seja da natureza. Daí que "they were regarded as eternal truths that man could try to discover but which he could not alter." (Hayek 1973, 73)

Contudo, com o advento do racionalismo construtivista, a crença exacerbada nos poderes da razão humana e a visão antropomórfica de que todas as instituições são, ou deveriam ser, fruto do desígnio humano, essa noção da lei como algo externo ao homem foi perdendo força. A legislação passou a ganhar espaço. Legislação, de acordo com Hayek, é "the deliberate making of law" (Hayek 1973, 72), fruto do desejo de que as práticas que regem o comportamento individual sejam resultado da avaliação racional 
humana e da vontade expressa do legislador, e tem como filosofia jurídica o positivismo legal (Hayek 1967c), profundamente ligado ao racionalismo construtivista.

Nessa perspectiva a distinção entre lei e legislação perde importância, na medida em que só possui legitimidade e efetividade o que for resultado do trabalho deliberado do legislador - e é essa qualidade que define o que é ou não justo. De acordo com Hayek, "the suggestion contained in this term [positivismo legal] that only deliberately made law is real law still provides the essential core of the positivist doctrine on which all its other assertions depend" (Hayek 1976a, 45), e, como consequência:

Yet the definition of law as the product of the will of the legislator leads not only to the inclusion in 'law' of all the expressions of the will of the legislator, whatever its content (...) but also to the view that content constitutes no significant distinction between different statements called law, and, in particular, that justice can in no sense be a determinant of what in fact is law but that it is rather the law which determines what is just. Contrary to the older tradition which had regarded justice as prior to law, and at least certain parts of law as limited by conceptions of justice, the contention that the lawgiver was the creator of justice became the most characteristic tenet of legal positivism. (Hayek 1976a, 48)

Ao lidar com a capacidade estatal de criar e impor leis, Hayek (2007, cap. 6) defende que a lei não deve ser usada para buscar fins específicos (até porque esse tipo de política, em um ambiente complexo, traz resultado imprevisíveis) ou para atingir determinado grupo restrito de pessoas. O que ele procura argumentar é que a rule of law deve prevalecer nas sociedades livres, de maneira a proporcionar aos participantes do mercado tanto a previsibilidade sobre quais serão as ações do governo quanto o espaço necessário para a busca do interesse próprio e o uso do conhecimento privado nisso. A rule of law é a situação em que a atuação do governo está bem delimitada por regras formais, estáveis e prévias, de modo que a ação individual possa ocorrer sobre um ambiente estável, em que é sabido pelos indivíduos que o poder coercitivo estatal não será utilizado de maneira imprevista, arbitrária e deliberada contra suas atividades. 
O papel da lei deve ser, na perspectiva hayekiana, o de fornecer uma plataforma geral, estável, previsível e anônima para a atuação dos agentes no processo de mercado, de maneira a se maximizar o uso social do conhecimento. Nesse sentido, Buchanan (2009) traça um paralelo entre os conceitos hayekianos de lei e legislação e os seus próprios. Segundo ele, o que Hayek pensava sobre lei pode ser comparado ao que, na teoria da escolha pública, é chamado de constituição, ao passo que legislação é o resultado que emerge da ação coletiva ordinária. Em outras palavras, constituição/lei fornece os limites da ação, ao passo que legislação pode ser pensada como ação pós (ou intra) constitucional.

A partir deste ponto de vista, pode-se entender que Hayek estabelece a distinção entre lei e legislação da seguinte maneira: lei é o estágio mais alto de delimitação da ação individual, ao estabelecer princípios mais gerais dentro dos quais as pessoas criam expectativas e tomam suas decisões corriqueiras. Por outro lado, legislação é o conceito que lida com estas ações mais comuns, que podem se alterar mais facilmente de acordo com as vicissitudes do cotidiano, com a dinâmica das relações humanas e suas consequências intencionais ou não, e com o crescimento do conhecimento.

\section{Considerações finais}

No artigo foi mostrado que o estudo das instituições por parte de Hayek decorreu dos questionamentos levantados pelo autor às suposições sobre conhecimento e equilíbrio usualmente feitas pelos economistas. Ao investigar problemas de conhecimento e coordenação, o austríaco se aprofundou cada vez mais na análise institucional sob uma perspectiva interdisciplinar, envolvendo contribuições da Economia, da Política e da Filosofia (Boettke 2000), e tendo por pano de fundo sua crítica ao racionalismo construtivista no debate do cálculo econômico socialista. Como parte da tentativa de se compreender a abordagem institucional hayekiana, foi proposta uma classificação de termos usados por aquele autor, com o propósito adicional de se facilitar o diálogo entre o institucionalismo hayekiano (e austríaco) e outras abordagens.

Os elementos levantados ao longo do ensaio explicitam que, em Hayek, as regras e instituições exercem uma influência sobre os indivíduos que vai muito além da mera restrição ao comportamento individual. É preci- 
samente ao estudar o papel que essas regras e instituições possuem, bem como sua relação com o desempenho do grupo que adere a elas, que Hayek argumenta contra as recomendações da corrente de pensamento que ele chama de racionalismo construtivista. $\mathrm{Na}$ visão do austríaco, as instituições estão intimamente ligadas à capacidade racional limitada dos indivíduos e ao comportamento e percepção de mundo deles, por um lado, e à utilização do conhecimento social total e cooperação anônima existente no mercado, de outro.

\section{Referências}

Boettke, Peter. 1990. "The Theory of Spontaneous Order and Cultural Evolution in the Social Theory of F. A. Hayek.” Cultural Dynamics 3 (1): $61-83$.

Boettke, Peter. 1998. "Economic Calculation: The Austrian Contribution to Political Economy." Advances in Austrian Economics 5: 131 - 158.

Boettke, Peter. 2000. The Legacy of Friedrich von Hayek. Cheltenham: Edward Elgar.

Boettke, Peter. 2000. "Which Enlightenment, Whose Liberalism? Hayek's Research Program for Understanding the Liberal Society.” In The Legacy of Friedrich von Hayek 1, by Peter Jay Boettke. Cheltenham: Edward Elgar.

Boettke, Peter. 2005. "Hayek and Market Socialism: Science, Ideology and Public Policy.” Economic Affairs 25 (4): $54-60$.

Boettke, Peter, Emily C. Schaeffer and Nicholas A. Snow. 2010. "The Context of Context: The Evolution of Hayek's Epistemic Turn in Economics and Politics.” Advances in Austrian Economics 14: 69 - 86.

Buchanan, James M. 2010. “The Constitutionalization of Money.” Cato Journal 30 (2): 251 - 258.

Butos, William N., and Roger G. Koppl. 2006. "Does the Sensory Order Have a Useful Economic Future?" Advances in Austrian Economics 9: 19 - 50.

Caldwell, Bruce J. 2004. Hayek’s Challenge: An Intellectual Biography of F. A. Hayek. Chicago: The University of Chicago Press.

De Andrade, Rogerio P. 2004. “On fallible knowledge.” Nova Economia 14 (1): 123 - 149.

Dequech, David. 2011. "Instituições e a Relação entre Economia e Sociologia.” Estudos Econômicos 41 (3): $599-619$.

Di Iorio, Francesco. 2010. "The Sensory Order and the Neurophysiological Basis of Methodological Individualism.” Advances in Austrian Economics 13: 179 - 209.

Ebenstein, Alan O. 2001. Friedrich Hayek: A Biography. New York: Palgrave.

Feijó, Ricardo. 2000. Economia e Filosofia na Escola Austríaca: Menger, Mises e Hayek. São Paulo: Nobel.

Feser, Edward. 2006. The Cambridge Companion to Hayek. Cambridge: Cambridge University Press.

Fleetwood, Steve. 1995. Hayek’s Political Economy: The Socio-Economics of Order. London: Routledge.

Fleetwood, Steve. 1997. “Hayek III: The Necessity of Social Rules of Conduct.” In Stephen F. Frowen. 1997.

Frowen, Stephen F. 1997. Hayek the Economist and Social Philosopher: a Critical Retrospective. London: Macmillan. 
Garrison, Roger, and Israel Kirzner. 1987. "Friedrich August von Hayek.” In The New Palgrave: A Dictionary of Economics, by John Eatwell, Murray Milgate, Peter Newman. London: Macmillan Press.

Garrison, Roger, and Norman P. Barry. 2014. Elgar Companion to Hayekian Economics. Cheltenham: Edward Elgar.

Gray, John N. 1984. Hayek on Liberty. London: Routledge.

Lewis, Paul A. 2014. "Hayek: From Economics as Equilibrium Analysis to Economics as Social Theory." In R. Garrison and N. P. Barry. 2014.

O'Driscoll, Gerald P. 1977. Economics as a Coordination Problem: The Contributions of Friedrich A. Hayek. Kansas City: Sheed Andrews and McMeel.

Soromenho, Jorge Eduardo de Castro. 1994. "Um Estudo Sobre as Origens da Crítica de Hayek ao Conceito de Equilíbrio." Tese Doutorado em Economia, Faculdade de Economia, Administração e Contabilidade. Universidade de São Paulo, São Paulo.

Vaughn, Karen I. 1999. "Hayek's Implicit Economics: Rules and the Problem of Order." Review of Austrian Economics $11(1-2): 129-144$.

Von Hayek, Friedrich August. 1937. "Economics and Knowledge.” In Friedrich A. Hayek. 1948a.

Von Hayek, Friedrich August. 1945. "The use of knowledge in society.” In Friedrich A. Hayek. 1948a.

Von Hayek, Friedrich August. 1946. "Individualism: True and False.” In Friedrich A. Hayek. 1948a.

Von Hayek, Friedrich August. 1948a. Individualism and Economic Order. Chicago: The University of Chicago Press.

Von Hayek, Friedrich August. 1948b. “The Meaning of Competition.” In Friedrich A. Hayek. 1948a.

Von Hayek, Friedrich August. 1960. The Constitution of Liberty. Chicago: The University of Chicago Press.

Von Hayek, Friedrich August. 1962. "Rules, Perception and Intelligibility.” In Friedrich A. Hayek. 1967b.

Von Hayek, Friedrich August. 1965. “Kinds of Rationalism.” In Friedrich A. Hayek. 1967b.

Von Hayek, Friedrich August. 1967a. "Notes on the Evolution of Systems of Rules of Conduct." In Friedrich A. Hayek. 1967b.

Von Hayek, Friedrich August. 1967b. Studies in Philosophy, Politics and Economics. Chicago: The University of Chicago Press.

Von Hayek, Friedrich August. 1967c. "The Results of Human Action but not of Human Design.” In Friedrich A. Hayek. 1967 b.

Von Hayek, Friedrich August. 1968. "The Confusion of Language in Political Thought.” In Friedrich A. Hayek. 1978.

Von Hayek, Friedrich August. 1969. “The Primacy of the Abstract.” In Friedrich A. Hayek. 1978.

Von Hayek, Friedrich August. 1970. "The Errors of Constructivism.” In Friedrich A. Hayek. 1978.

Von Hayek, Friedrich August. 1973. Law, Legislation and Liberty: A New Statement of the Liberal Principles of Justice and Political Economy. Vol. 1: "Rules and Order." London: Routledge.

Von Hayek, Friedrich August. 1976a. Law, Legislation and Liberty: A New Statement of the Liberal Principles of Justice and Political Economy. Vol. 2: "The Mirage of Social Justice." London: Routledge.

Von Hayek, Friedrich August. 1976b. The Sensory Order. Chicago: The University of Chicago Press.

Von Hayek, Friedrich August. 1978. New Studies in Philosophy, Politics, Economics and the History of Ideas. London: Routledge.

Von Hayek, Friedrich August. 1979. Law, Legislation and Liberty: A New Statement of the Liberal Principles of Justice and Political Economy. Vol. 3: "The Political Order of a Free People.” London: Routledge. 
Von Hayek, Friedrich August. 1982. Consolidated Preface to One-Volume Edition Law, Legislation and Liberty: A New Statement of the Liberal Principles of Justice and Political Economy, by Friedrich A. Hayek. London: Routledge.

Von Hayek, Friedrich August. 1988. The Fatal Conceit: The Errors of Socialism. The Collected Works of Friedrich A. Hayek 1. Chicago: The University of Chicago Press.

Von Hayek, Friedrich August. 2007. The Road to Serfdom: Text and Documents. The Collected Works of Friedrich A. Hayek 2. Chicago: The University of Chicago Press.

Wood, John Cunningham, and Ronald N. Woods. 1991. Friedrich A. Hayek: Critical assessments. London: Routledge. 